\title{
Application of 3D Navigation and Vascular Reconstruction Technology in Precision Resection of Lung Segment
}

\author{
Liusheng $\mathrm{Wu}^{1,2}$, Xiaoqiang $\mathrm{Li}^{1,2, *}$ \\ ${ }^{1}$ Peking University Shenzhen Hospital, Clinical College of Anhui Medical University, Shenzhen, Guangdong518036, China \\ ${ }^{2}$ Department of thoracic surgery, Peking University Shenzhen Hospital, Shenzhen, Guangdong518036, China
}

\begin{abstract}
With the rapid development of imaging technology and the improvement of people's health awareness, more early lung cancers are found. For very early lung cancer (mainly manifested as ground glass nodules), lobectomy is still the standard surgical treatment for lung cancer, and segmental resection has been used clinically as a treatment method. 3D navigation is an effective tool for precise lung segment and sub-segment resection. Three-dimensional vascular reconstruction technology is an advanced imaging technology. Accurate segment resection under thoracoscopy is considered the best surgical treatment for early stage lung cancer. It is difficult to accurately estimate the morphology, nature and other characteristics of the tumor and its surroundings by two-dimensional imaging alone. Therefore, the precise resection operation adds great difficulty and risk to the surgeon. In recent years, 3D navigation and vascular reconstruction techniques have been widely developed and applied in the medical field, and they are rarely used in thoracic surgery. This article has conducted a more in-depth study on the application of 3D navigation technology and vascular reconstruction technology in precision lung resection, and has achieved certain research results.
\end{abstract}

\section{Introduction}

In the past 20 years, with the development of biomedicine and the rise of evidence-based medicine and humanistic medicine, the concept of surgery has been constantly changing. Simply paying attention to the physical effects of surgical treatment is no longer the only goal pursued by surgical operations. The traditional empirical surgical model is changing to a modern precision surgical model. In 2006, foreign countries first proposed the concept of "precision surgery" and applied it to the clinical practice of hepatobiliary surgery [1]. Precision surgery is centered on surgery. It seeks to find the precise balance of the three surgical elements of removing the focus, protecting the organs, and controlling the damage as a strategy [2]. It covers disease evaluation, clinical decision-making, surgical planning, surgical operations and perioperative management. The whole process of surgical practice [3]. It is a brand-new surgical concept and technical system, which has been applied to many clinical medicine fields such as neurosurgery, hepatobiliary surgery, breast surgery, otolaryngology head and neck surgery and so on [4]. Lung surgery is unique. During the operation, the lung tissue has a certain degree of collapse and centripetal or rotational deformation, and in the thoracoscopic view, the operation field cannot be fully observed due to the limitation of the visual field [5]. For this reason, in order to be safe and effective, it is very important to understand the branch form of the blood vessel before surgery. In this way, people can non-invasively observe the branching pattern of blood vessels and bronchus [6].

\section{3D navigation and vascular reconstruction technology}

\subsection{D navigation technology}

The invention and application of computer-assisted navigation system can change the traditional surgical operation mode in the medical field, and make the surgical treatment process more intelligent, minimally invasive and precise, thereby reducing the trauma caused during the operation, improving the safety of the operation, and reducing complication [7]. The computeraided navigation system is used during the operation, that is, the operation process and images are passed through the computer image processing system to realize the visualization of the operation process and the threedimensional space, thereby improving the accuracy of the operation [8].

$3 \mathrm{D}$ navigation technology is a three-dimensional positioning system based on the global satellite positioning system [9]. The workflow used in the medical field is to perform preoperative digital scans of patients and input the obtained image information to a computer workstation (media) [10]. The workstation can perform three-dimensional reconstruction, image registration, and image fusion according to the patient's

\footnotetext{
* Corresponding author:_dr.lixiaoqiang@gmail.com
} 
preoperative situation to establish a virtual coordinate space of the patient's body before surgery [11]. Doctors can perform related preoperative simulation operations on the basis of virtual images constructed by computers, and use the system's infrared camera to dynamically track surgical instruments during actual surgery, locate the patient's surgical site, and determine the patient's relevant spatial positioning data. The doctor combines the two-dimensional and three-dimensional data of the patient with virtual images and displays the positioning images in real time. According to the high-level image display screen, the surgical process can be fully controlled, including the location of the lesion, the surgical incision approach and the approach. The angle and depth of the disease can be treated as quickly and accurately as possible, reducing the trauma caused by the operation to the patient, shortening the operation time, and reducing postoperative complications [12].

\subsection{Vascular reconstruction technique}

Three-dimensional CT bronchial vascular reconstruction technology refers to the thin-layer enhanced CT scan of the patient, the obtained two-dimensional image is extracted and segmented by software to construct threedimensional geometric figures, and a three-dimensional digital model is formed through computer visualization technology to locate an imaging technique for the location of the lesion [13]. This technology performs three-dimensional $\mathrm{CT}$ bronchial vascular reconstruction for patients with early lung cancer, which can reconstruct three-dimensional images of the three major structures of the lungs (including bronchi, arteries, and veins), clearly showing the anatomical structure of the lung segment and the variation of the lung bronchi and blood vessels [14]. Under the circumstances, the lung segment to which the tumor belongs is clear, and the image can be rotated $360^{\circ}$ freely, which can quickly and intuitively identify the anatomical structure of the target lung segment, which helps to assess the difficulty of the operation. We can plan the surgical path and the scope of surgical resection through continuous segmentation, layer-by-layer separation, etc., and we can develop individualized surgical plans for patients based on the anatomical characteristics of the target lung segment of the lesion, and then achieve preoperative planning, navigation, simulation, eduction and the purpose of intraoperative control anatomy [15]. In recent years, three-dimensional CT bronchial vascular reconstruction technology has been widely used in surgical operations such as hepatobiliary surgery and urological surgery, and has achieved remarkable results. At present, threedimensional CT bronchial vascular reconstruction software commonly used in my country includes IQQA, Osiri $\mathrm{X}$ and so on [16].

\section{Materials and Methods}

\subsection{Clinical information}

The patient with chest CT pulmonary nodules in this study, male, age 41 years old. The thickness of CT scan is $1.25 \mathrm{~mm}$. In this scan, two nodules were identified. As shown in Figure 1.



Fig. 1. Morphological nodule positions extracted from CT scan

\subsection{Method}

All patients underwent chest CT bronchial CTA examination and GE Revolution CT scan. An indwelling needle was placed in the median vein of the right elbow, and a non-ionic contrast agent iopromide $(370 \mathrm{mg} / \mathrm{ml})$ was used. Adopt the "bolus injection test method", that is, select the clearly displayed level of the pulmonary artery and aorta, and perform a low-dose bolus injection test with $30 \mathrm{ml}$ contrast medium and the same amount of normal saline to obtain the peak time of the pulmonary artery and aorta; then according to the peak time and scan time of the pulmonary artery and aorta, the contrast injection dose and scan delay time are accurately calculated.

Scanning scheme: tube voltage $120 \mathrm{kV}$, tube current $200 \mathrm{~m}$, collimator $128 \mathrm{~mm} \times 0.625 \mathrm{~mm}$, matrix $512 \times 512$, reconstructed thin layer images with a thickness of 1.0 $\mathrm{mm}$ and an interval of $1.0 \mathrm{~mm}$; scanning direction: topdown. The thin-layer image is transmitted to the Philips Nebula Space Workstation for post-processing such as multiplanar reconstruction (MPR), maximum intensity projection (MIP), and volume rendering (VR). The surgeon enters the enhanced CT DICOM data of the patient's chest into Deep Insight software and runs it. After image loading processing, tracheal threshold calculation, tracheal extraction, blood vessel extraction, lung nodule extraction, blood vessel staining and other steps, the 3D lung bronchus and movement are reconstructed. Model of veins and form 3D animated images. During the operation, compare the 3D lung bronchus and blood vessel imaging with the actual anatomical results to observe the $3 \mathrm{D}$ navigation effect. 

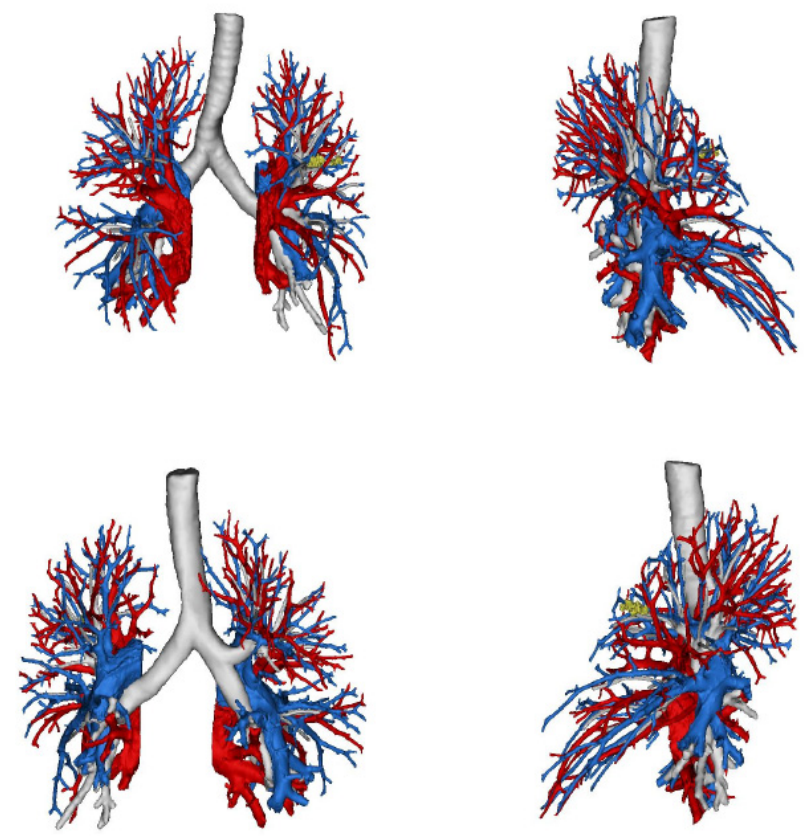

Fig. 2. Overview of the three-dimensional model of small lung nodules

Among them, red represents veins, blue represents arteries, white represents trachea, and yellow represents nodules.

\section{Discussion}

In the process of performing thoracoscopic precision segmentectomy for patients with early-stage lung cancer, the preoperative three-dimensional CT bronchial vascular reconstruction image should be placed next to the thoracoscopic display. Carry out real-time navigation and comparison during the operation to detect and correct the deviation of the operation in time; adjust and optimize the surgical plan at any time, improve the accuracy and safety of the operation, reduce the surgical trauma of the patient, and reduce the incidence of postoperative complications. Some scholars conducted a study on 68 patients with early stage lung cancer who underwent thoracoscopic precision segmentectomy. The results showed that the time and difficulty of the operation for patients who received three-dimensional CT bronchial vascular reconstruction before surgery were significantly better than those before surgery.

When using software to rebuild blood vessels, some small arteries and veins may be confused, and you need to pay attention to screening at this time. Each software has its own advantages in reconstruction. At present, mimics software is widely used and easier to obtain. It has been updated to version 22.0. Mimics, incool and other software use intersegment veins to divide lung segments, and IQQA software uses trachea as the core to divide intersegment planes. Because the lung segments are actually three-dimensional cones, some lung segments are not divided into each other in a straight line. In more cases, adjacent lung segments are interlaced with each other. Ventilation-collapse method, fluorescence, and pure oxygen expansion are used. When searching for the plane between segments, the boundary of the broken line will appear. Therefore, the reconstruction result of the software does not completely reflect the division of the plane between segments. Clinicians need to consider the reconstruction result and the boundary between segments during the operation. Compared with the veins, the pulmonary artery has less variation, and its shape is accompanied by the trachea. This feature can be used for mutual confirmation during reconstruction and surgery. If the pulmonary veins are disconnected too much, postoperative hemoptysis may occur. During segmentectomy, the intersegmental veins can be mutually confirmed with the intersegmental plane. After the branches of the veins between the main segments are disconnected, the treatment of the branches of the veins between the remaining segments can be weakened. Clinicians also need to pay attention to the transformation between intrasegmental veins and intersegmental veins. The intersegmental vein during segmental resection will change its role to the internal segmental vein during combined lung segmental or combined subsegmental resection. After 3D-CTBA reconstruction, based on the direction of tracheal blood vessels, clinicians can re-examine the surgical target area based on the reconstruction results and design the preoperative plan more accurately.

There are high technical requirements for segmentectomy. The pulmonary blood vessels and bronchi are buried in the lung tissue, and there are more anatomical variants of the pulmonary blood vessels and bronchi. Surgical injury can lead to postoperative hemoptysis, lung tissue leakage, infection, and atelectasis. In particular, pulmonary vein injuries can be complicated by severe hemoptysis, even requiring reoperation. Apply 3D stereo modeling to reconstruct the pulmonary artery, vein and bronchi to form a 3D stereo model, and display it in the form of 3D stereo animation. It can observe whether the arteries, veins, and bronchi are deformed and deformed, and accurately judge the nodules. The attribution of lung segments, the relationship between lung nodules, blood vessels and bronchi. It can provide the best surgical plan to achieve precise lung segment resection. At present, a few domestic hospitals use professional commercial 3D navigation software, which is expensive and complicated to operate, which is not conducive to popularization. Deep Insight is a 3D navigation software specially designed for thoracic surgery segment resection in my country. It has comprehensive functions and easy operation. It can be operated by the surgeon on its own and has a broad application prospect.

Since the pulmonary segmental veins walk between the lung segments to collect the venous return from adjacent pulmonary segments, the branches of the pulmonary segment should be preserved as much as possible during the operation to avoid obstacles to the venous return of adjacent pulmonary segments. The right upper pulmonary vein branches towards up to 4 types. Using 3D navigation, you can clearly understand the pulmonary vein classification and branch direction, so as to select the appropriate anatomical part. In one case, 3D 
navigation showed that the posterior trachea of the left upper lung apex was closely adjacent to the posterior pulmonary vein branch. The operation was carried out carefully to avoid vein damage. Similarly, pulmonary artery branches and pulmonary bronchi may also originate from adjacent lung segments or co-stems. Intraoperative 3D navigation can avoid accidental injury.

\section{Conclusion}

3D navigation and vascular reconstruction technology has been widely used in clinical practice. Its intuitive user interface and high-performance processing capabilities enable surgeons to observe various images generated in real time. Image segmentation and sculpting tools can extract and remove related anatomical structures, color and transparency adjustments are also used to provide the best $3 \mathrm{D}$ vision. Although this technology is still in its infancy, its benefits are obvious. It can guide medical staff to learn new technologies to improve the quality of surgical training and theoretical research. However, the current 3D reconstruction navigation technology is still limited to static navigation. In the future, artificial intelligence will be combined to develop tools for dynamic navigation during surgery, which will further improve the efficiency and accuracy of surgery.

\section{References}

1. J. Yang, S.P. Cai, J.L. Huang, et al. Application of digital 3D printing navigation template in tibia bone tumor surgery. China Digital Medicine, 2020, 15(10):122-124.

2. Z. Chai, L.L. Ren. Analysis of the short-term and long-term curative effect of 3D printing navigation template assisted by personalized non-restrictive tumor prosthesis reconstruction on malignant tumors around the knee join. Practical Journal of Cancer, 2020, 35(05):867-870.

3. Y.F. Yang, Z.Y. Wang. The application of threedimensional CT bronchial vascular reconstruction technology in thoracoscopic precision segmentectomy. Contemporary Medical Review, 2020, 18(07):20-22.

4. Y.G. Liu, Y.H. Sun, W.Q. Tao, et al. Application of spiral CT three-dimensional vascular reconstruction technique in venous erectile dysfunction. Journal of Guangxi Medical University, 2018, 35(08):11391140.

5. X.F. Lin, S.Y. Zheng. The application progress of hybrid coronary artery revascularization technology in the surgical treatment of coronary heart disease. Guangdong Medical Journal, 2017, 38(06):967-969.

6. X.J. Li, L. Zhang, Z. Tang, et al. The clinical application of three-dimensional reconstruction technology in thoracoscopic lung resection. Journal of Bengbu Medical College, 2020, 45(10):13631366.
7. L. Chen, J. Wang, W.B. Wu, et al. Technical process and quality control of thoracoscopic precision segmentectomy. Chinese Journal of Clinical Thoracic and Cardiovascular Surgery, 2019, 26(01):21-28.

8. J.B. Xie, P.F. Chen, T.B. Yang, et al. Application of 3D-CTBA technology in precision segmentectomy for small pulmonary nodules. Journal of Putian University, 2018, 25(05):23-27.

9. S.L. Zhang, B. Zheng, G.B. Xu, et al. Application of combined dimension reduction method in Da Vinci robot-assisted precision segmentectomy. Chinese Electronic Journal of Thoracic Surgery, 2019, 6(02):139- 140 .

10. W.B. Wu, X.F. Xu, W. Wen, et al. (2016) Threedimensional computed tomography bronchography and angiography in the preoperative evaluation of thoracoscopic segmentectomy and subsegmentectomy. J Thorac Dis, 8(Suppl 9): S710S715.

11. T. Nagashima, K. Shimizu, Y. Ohtaki, et al. (2015) An analysis of variations in the bronchovascular pattern of the right upper lobe using threedimensional CT angiography and bronchography. Gen Thorac Cardiovasc Surg, 63(6): 354-60.

12. M. Sato, M. Omasa, F. Chen, et al. (2014) Use of virtual assisted lung mapping (VAL-MAP), a bronchoscopic multispot dye-marking technique using virtual images, for precise navigation of thoracoscopic sublobar lung resection. Journal of Thoracic \& Cardiovascular Surgery, 2014, 147(6):1813.

13. F. Yao, J. Wang, J. Yao, et al. (2017) Threedimensional image reconstruction with free opensource Osiri X software in video-assisted thoracoscopic lobectomy and segmentectomy. International Journal of Surgery(London,England), (39):16-22.

14. H. Nitanda, R. Taguchi, A. Yanagihara, et al. (2019) Surgical Outcome of Sublobar Resection in Highrisk Patients with Non-small Cell Lung Cancer. Kyobu geka. The Japanese journal of thoracic surgery, 72(1):17-22.

15. L. Xue, H. Fan, W.D. Shi, et al. (2018) Preoperative 3-dimensional computed tomography lung simulation before video-assisted thoracoscopic anatomic segmentectomy for ground glass opacity in lung. Journal of Thoracic Disease, 10(12):65986605.

16. Ha KJ, Yun JK, Lee GD, et al. (2018) Surgical Outcomes of Radiographically Noninvasive Lung Adenocarcinoma according to Surgical Strategy: Wedge Resection, Segmentectomy, and Lobectomy. The Korean journal of thoracic and cardiovascular surgery, 51(6):376-383. 